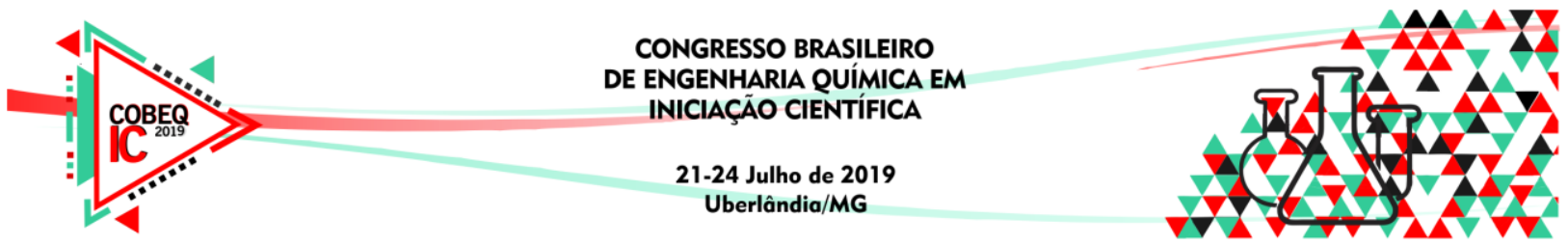

\title{
HIDRÓLISE DE ÓLEO DE SOJA DEGOMADO CATALISADO POR LIPASE DE PSEUDOMONAS FLUORESCENS LIVRE: AVALIAÇÃO DE REUSO DE ÁGUA E ENZIMA.
}

\author{
R. F. C. BENTO ${ }^{1}$ e P. W. TARDIOLI ${ }^{1}$ \\ ${ }^{1}$ Universidade Federal de São Carlos, Departamento de Engenharia Química, São Carlos, SP \\ ${ }^{2}$ Universidade Federal de São Carlos, Departamento de Engenharia Química, São Carlos, SP \\ E-mail para contato: renatofcbento@hotmail.com
}

\begin{abstract}
RESUMO - Com enormes preocupações relacionadas aos combustíveis fósseis, tanto em caráter de futura escassez quanto ambiental, o biodiesel, por ser biodegradável e renovável, prova-se uma alternativa interessante para suprir essa demanda energética. Dentre os processos para sua obtenção, o mais consolidado, conhecido como rota química, resume-se na transesterificação entre óleos e álcoois, gerando glicerol e biodiesel, na presença de catalisadores básicos, e dentre suas desvantagens, cita-se a necessidade de pré-tratamento de acidez da matéria-prima e do $\mathrm{pH}$ dos efluentes para descarte, reação com alta demanda de álcool e ausência total de água, dificuldade de recuperação do glicerol, altas temperaturas de operação e corrosão do sistema de operação. Por estas razões, a hidroesterificação catalisada por enzimas, consistindo na hidrólise de óleos com água, formando glicerol, água e ácidos graxos livres, em sequência da esterificação deste último com álcoois, formando água e biodiesel, é uma rota candidata ao aperfeiçoamento da produção de biodiesel, pois opera em condições moderadas de temperatura, não necessita de pré-tratamento de acidez da matéria prima, e o glicerol produzido é mais puro e de mais fácil recuperação. Este trabalho descreve o perfil de conversão da hidrólise de óleo de soja degomado catalisada por lipase de Pseudomonas fluorescens (LPF) livre, e a avaliação do reuso da água e lipase em sucessivas hidrólises realizadas no tempo de máxima conversão.
\end{abstract}

\section{INTRODUÇÃO}

Lipases são enzimas de origem animal, vegetal ou microbiana, que convertem cataliticamente triglicerídeos em ácidos graxos, glicerol e mono e di-glicerídeos. Podem ser classificadas, quanto a sua especificidade, em: lipases ácido-graxo específicas (atuam apenas no carbono 9 do triglicerídeo), 1-3 específicas (catalisam apenas os ácidos graxos das posições 1 e 3 dos triacilgliceróis) e não específicas hidrolisam moléculas de triacilgliceróis, produzindo ácidos graxos livres, glicerol, monoacilgliceróis e diacilgliceróis como intermediários. Para otimizar a produção de biodiesel, a lipase selecionada deve converter todas as três formas de glicerídeos, ou seja, ser não-específica, e um dos maiores obstáculos em aplicação industrial de enzimas é o seu alto custo de produção (Pourzolfaghar et al.,2016). 


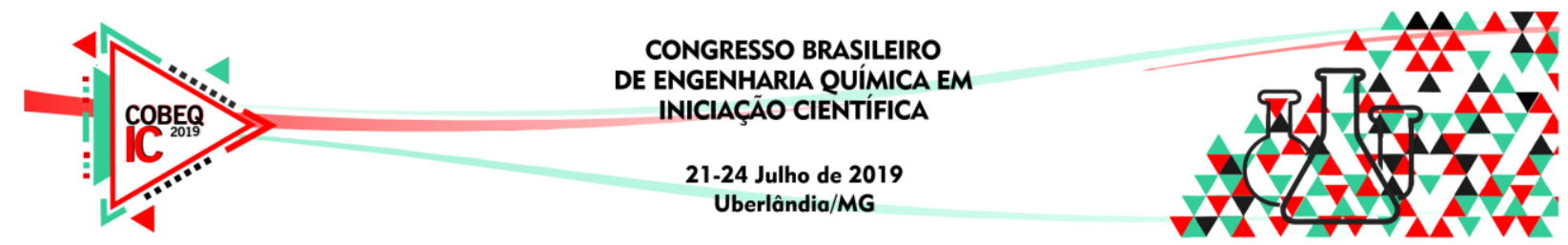

Segundo Pourzolfaghar et al., a hidroesterificação é um processo integrado de hidrólise, seguida de esterificação, para a produção de biodiesel, conforme ilustrado na Figura 1. Diferente da maneira convencional, este método catalisado por enzimas não possui formação de sabão, trabalha em condições próximas à ambiente, e produz um glicerol mais puro, de maior valor agregado. Outra vantagem do processo é a possibilidade de trabalhar com matérias-primas brutas, como óleos e gorduras sem tratamentos prévios de acidez, os quais possuem um baixo preço de mercado. (Vescovi et al.,2016)

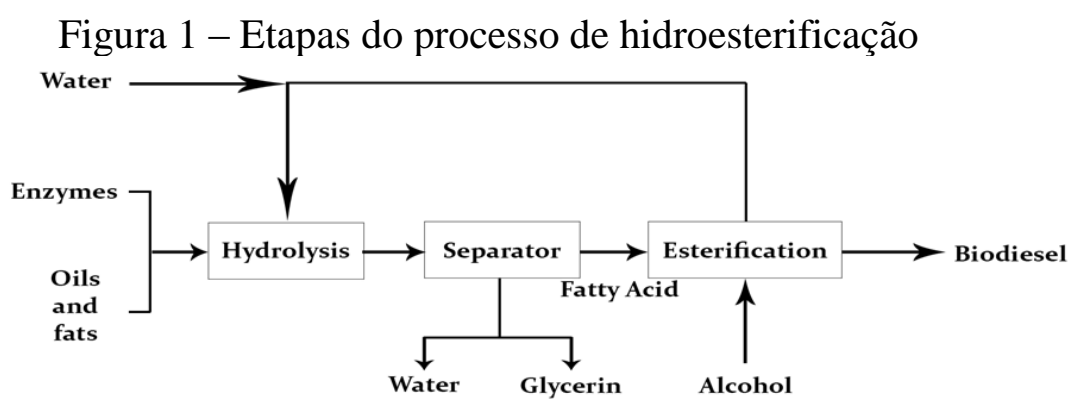

Segundo a Associação Brasileira das Indústrias de óleos vegetais, ABIOVE, o óleo de soja degomado é obtido a partir do pensamento da semente de soja, sendo uma matéria prima de baixo valor de mercado, e abundante no cenário econômico brasileiro atual.

Este trabalho é continuação de um trabalho desenvolvido na disciplina de Desenvolvimento de Processos Químicos de 2017, ofertada pelo Departamento de Engenharia da UFSCar, em que foi determinado as condições experimentais para a hidrólise de óleo de soja degomado. Cita-se: seleção de enzima (Lipase de Pseudomonas fluorescens), temperatura de operação $\left(35^{\circ} \mathrm{C}\right)$, tipo e agitação de empelidor (pás triplas, $900 \mathrm{rpm}$ ), carga enzimática (100U/g de óleo de soja degomado), razão de massa de óleo e água no reator $(1: 0,5)$, e índice de Saponificação do óleo de soja degomado (IS $=189,82 \mathrm{mg} \mathrm{KOH} / \mathrm{g}$ de óleo de soja degomado).

\section{MATERIAIS E MÉTODOS}

\subsection{Perfil de conversão com o tempo}

Sabe-se que estudar e entender como a cinética química se desenvolve ao longo do tempo é um fator que permite avaliar a viabilidade de aplicação de um processo industrial, uma vez que atingir uma determinada taxa de conversão pode demandar um elevado tempo de reação ou mesmo ser termodinamicamente inatingível (Davis e Davis, 2003). Dessa forma, conhecer o ponto ótimo de operação é fundamental para um processo industrial.

O experimento foi realizado com a finalidade de acompanhar o aumento da conversão da hidrólise de óleo de soja degomado catalisado pela lipase de Pseudomonas fluorescens livre ao longo do tempo em dois reatores batelada sob as mesmas condições, objetivando encontrar o tempo necessário para atingir a máxima conversão possível. Após o início da reação, buscou-se retirar alíquotas de $2 \mathrm{~mL}$ nos tempos de 0,5;1; 2 e 3 horas, e em seguida, de 3 em 3 horas para titulação (além da alíquota controle, antes da reaão iniciar). As alíquotas de $2 \mathrm{~mL}$ foram retiradas com pipeta volumétrica de $5 \mathrm{~mL}$, com a extremidade da ponteira cortada para garantir proporção equivalente de água:óleo da alíquota e do reator. 


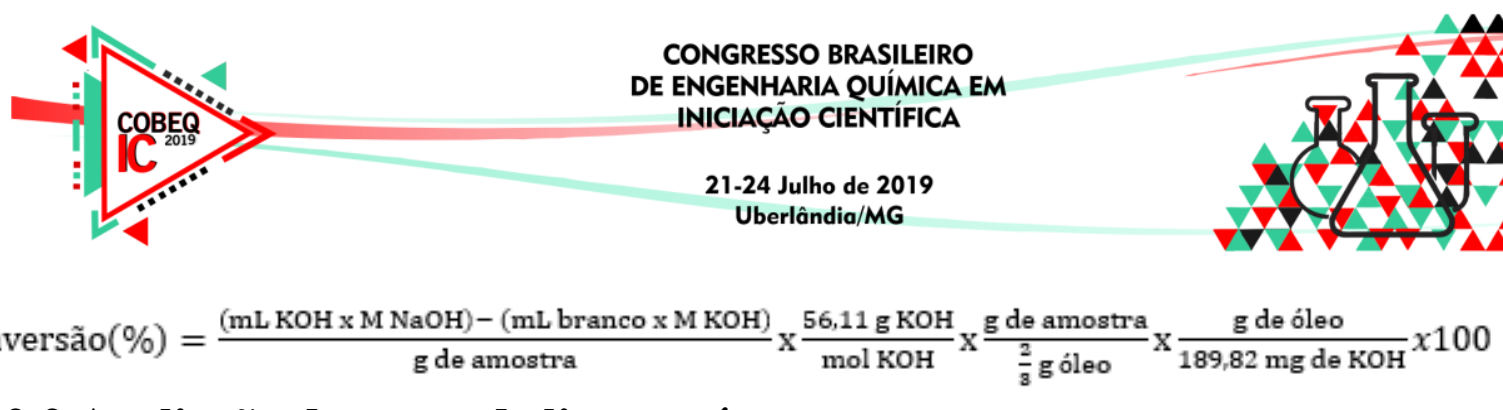

\subsection{Avaliação do reuso de lipase e água}

É sabido que na rota enzimática de produção de biodiesel, o maior custo é associado ao elevado preço dos biocatalisadores (Rosset et al., 2019). Por essa razão, foi realizado uma avaliação do reuso sucessivo da fase pesada do produto de uma batelada de hidrólise de óleo de soja degomado catalisado por lipase de Pseudomonas fluorescens, composto por água, lipase, e glicerol, este último que fica mais concentrado a medida que cada reuso é feito.

O tempo de cada batelada foi o equivalente ao tempo de máxima conversão, obtido pelo perfil de conversão ao longo do tempo, e ao fim de cada batelada, desligou-se banho de laboratório e agitação, e esperou-se 3,5 horas para ocorrer a decantação para permitir a retira de ácidos graxos livres e do óleo de soja degomado que não reagiu. O cálculo de conversão foi realizado com a Equação 1, também utilizada no experimento do perfil de conversão ao longo do tempo.

\section{RESULTADOS E DISCUSSÕES}

\subsection{Perfil de conversão com o tempo}

De acordo com o procedimento experimental descrito, tomou-se notas, no momento da retirada das alíquotas de $2 \mathrm{~mL}$ para titulação, de suas massas e do tempo decorrido de reação (sendo o momento inicial, ou tempo zero, o instante em que a lipase foi introduzida nos reatores). Tomou-se nota, também, da concentração e do volume da solução de hidróxido de sódio necessária para a titulação das amostras, e a partir da Equação 1, foi possível calcular a conversão da hidrólise de óleo de soja degomado catalisado pela lipase de Pseudomonas fluorescens livre em função do tempo de reação. Os dados obtidos são apresentados nas Tabelas 1 e 2 a seguir, referentes aos dois reatores em que se realizou o experimento, e o gráfico Conversão (\%) vs Tempo (h) com um ajuste de curva e desvio dos dados obtidos, apresentado na figura 2:

Tabela 1 - Dados obtidos experimentalmente para o Reator 1 referente às titulações de alíquotas obtidas, ao longo do tempo, da hidrólise de óleo de soja degomado

\begin{tabular}{|c|c|c|c|c|c|c|c|c|}
\hline Dia & Horário & $\begin{array}{c}\text { Tempo } \\
\text { reação }(\mathrm{h})\end{array}$ & $\begin{array}{c}\text { Massa total } \\
\text { água + óleo } \\
\text { reator }(\mathrm{g})\end{array}$ & $\begin{array}{c}\text { Massa } \\
\text { amostra (g) }\end{array}$ & $\begin{array}{c}{[\mathrm{KOH}]} \\
(\mathrm{mol} / \mathrm{L})\end{array}$ & $\begin{array}{l}\text { Vol. } \\
\mathrm{KOH} \\
(\mathrm{ml})\end{array}$ & $\begin{array}{l}\text { Mol. } \mathrm{KOH} / \mathrm{g} \text { de } \\
\text { mistura }(\mathrm{mol} / \mathrm{g})\end{array}$ & Conversão (\%) \\
\hline \multirow{7}{*}{1} & \multirow{2}{*}{$11: 10$} & \multirow{2}{*}{0,00} & \multirow{2}{*}{151,2458} & 1,8440 & 0,01882 & 0,40 & \multirow{2}{*}{$4,07 \mathrm{E}-06$} & \multirow{2}{*}{0,00} \\
\hline & & & & 1,8572 & 0,01882 & 0,40 & & \\
\hline & $11: 45$ & 0,58 & 147,5446 & 1,6497 & 0,01882 & 46,50 & $5,30 \mathrm{E}-04$ & 23,34 \\
\hline & $12: 30$ & 1,33 & 145,8949 & 1,6632 & 0,24086 & 6,00 & $8,69 \mathrm{E}-04$ & 38,35 \\
\hline & $14: 00$ & 2,83 & 144,2317 & 1,6311 & 0,24086 & 7,60 & $1,12 \mathrm{E}-03$ & 49,58 \\
\hline & $15: 00$ & 3,83 & 142,6006 & 1,6359 & 0,24086 & 8,00 & $1,18 \mathrm{E}-03$ & 52,05 \\
\hline & $18: 00$ & 6,83 & 140,9647 & 1,7109 & 0,24086 & 12,50 & $1,76 \mathrm{E}-03$ & 77,85 \\
\hline \multirow{4}{*}{2} & $09: 40$ & 22,50 & 139,2538 & 1,6829 & 0,24086 & 14,40 & $2,06 \mathrm{E}-03$ & 91,20 \\
\hline & $12: 50$ & 25,67 & 137,5709 & 1,7026 & 0,24086 & 15,00 & $2,12 \mathrm{E}-03$ & 93,91 \\
\hline & \multirow{2}{*}{$15: 50$} & \multirow{2}{*}{28,67} & \multirow{2}{*}{135,8683} & 1,6159 & 0,24086 & 14,80 & \multirow{2}{*}{$2,11 \mathrm{E}-03$} & \multirow{2}{*}{93,57} \\
\hline & & & & 1,8457 & 0,24086 & 15,50 & & \\
\hline
\end{tabular}




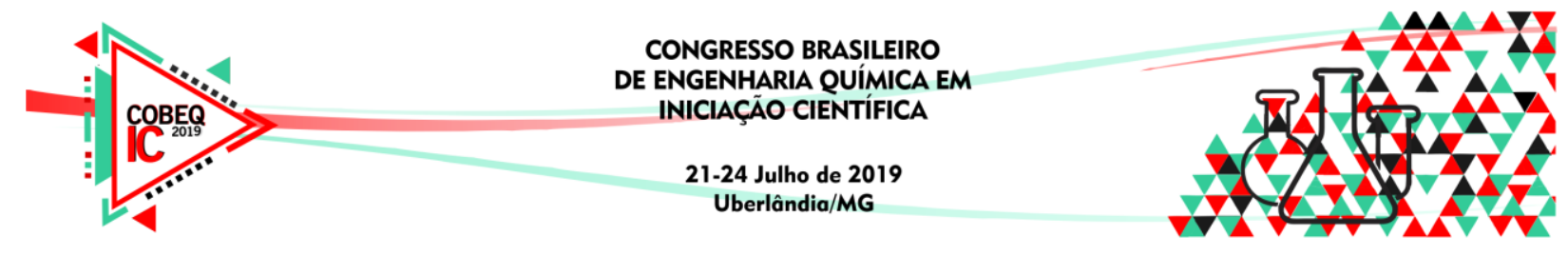

Tabela 2 - Dados obtidos experimentalmente para o Reator 2 referente às titulações de alíquotas obtidas, ao longo do tempo, da hidrólise de óleo de soja degomado

\begin{tabular}{|c|c|c|c|c|c|c|c|c|}
\hline Dia & Horário & $\begin{array}{c}\text { Tempo } \\
\text { reação }(\mathrm{h})\end{array}$ & $\begin{array}{c}\text { Massa total } \\
\text { água + óleo } \\
\text { reator }(\mathrm{g})\end{array}$ & $\begin{array}{c}\text { Massa } \\
\text { amostra (g) }\end{array}$ & $\begin{array}{c}{[\mathrm{KOH}]} \\
(\mathrm{mol} / \mathrm{L})\end{array}$ & $\begin{array}{l}\text { Vol. } \\
\mathrm{KOH} \\
(\mathrm{ml})\end{array}$ & $\begin{array}{c}\text { Mol. } \mathrm{KOH} / \mathrm{g} \mathrm{de} \\
\text { mistura (mol/g) }\end{array}$ & Conversão (\%) \\
\hline \multirow{7}{*}{1} & \multirow{2}{*}{$11: 10$} & \multirow{2}{*}{0,00} & \multirow{2}{*}{150,8868} & 1,8007 & 0,01882 & 0,40 & \multirow{2}{*}{$4,03 E-06$} & \multirow{2}{*}{0,00} \\
\hline & & & & 1,8881 & 0,01882 & 0,40 & & \\
\hline & $11: 45$ & 0,58 & 147,1980 & 1,9039 & 0,24086 & 5,90 & $7,46 \mathrm{E}-04$ & 33,09 \\
\hline & $12: 30$ & 1,33 & 145,2941 & 1,8461 & 0,24086 & 7,10 & $9,26 \mathrm{E}-04$ & 41,07 \\
\hline & $14: 00$ & 2,83 & 143,4480 & 1,7900 & 0,24086 & 8,20 & $1,10 \mathrm{E}-03$ & 48,92 \\
\hline & $15: 00$ & 3,83 & 141,6580 & 1,9142 & 0,24086 & 9,20 & $1,16 \mathrm{E}-03$ & 51,33 \\
\hline & $18: 00$ & 6,83 & 139,7438 & 1,9355 & 0,24086 & 11,40 & $1,42 \mathrm{E}-03$ & 62,90 \\
\hline \multirow{4}{*}{2} & $09: 40$ & 22,50 & 137,8083 & 1,9182 & 0,24086 & 15,90 & $2,00 \mathrm{E}-03$ & 88,52 \\
\hline & $12: 50$ & 25,67 & 135,8901 & 1,9693 & 0,24086 & 15,90 & $1,94 \mathrm{E}-03$ & 86,23 \\
\hline & \multirow{2}{*}{$15: 50$} & \multirow{2}{*}{28,67} & \multirow{2}{*}{133,9208} & 1,8963 & 0,24086 & 15,90 & \multirow{2}{*}{$2,03 E-03$} & \multirow{2}{*}{89,87} \\
\hline & & & & 1,9301 & 0,24086 & 16,30 & & \\
\hline
\end{tabular}

Com as conversões em função do tempo para ambos os reatores, foi possível plotar um gráfico Conversão (\%) vs Tempo (h) com um ajuste de curva e desvio dos dados obtidos, apresentado na figura 2 a seguir:

Figura 2 - Gráfico de conversão (\%) da reação de hidrólise de óleo de soja degomado catalisado pela lipase de Pseudomonas fluorescens em função do tempo de reação(h)

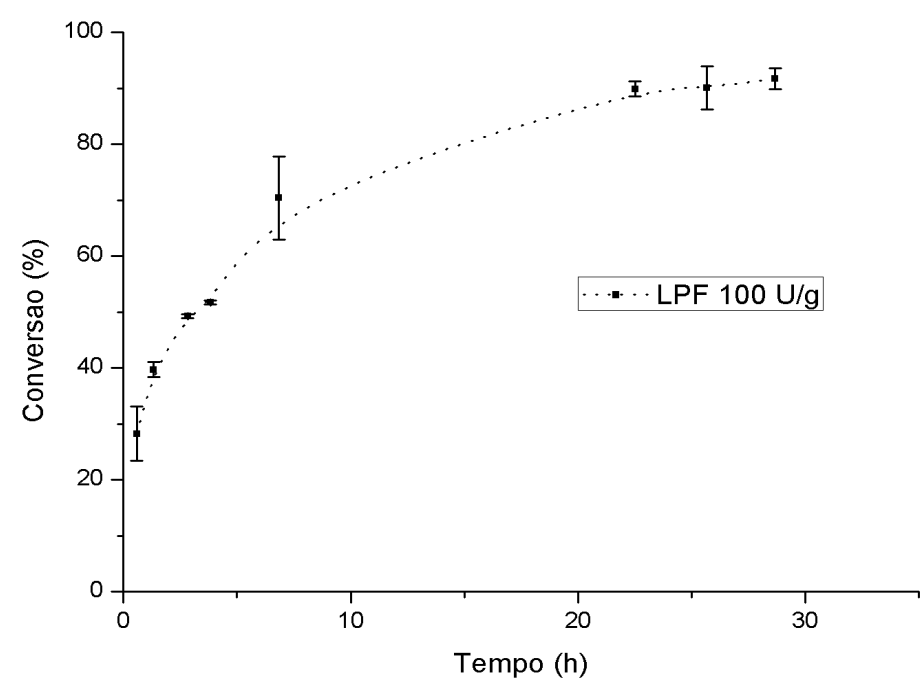

Segundo Shuler e Kargi, a cinética enzimática de um único substrato possui comportamento regido pelo modelo de Michaelis-Menten. A partir da Figura 2, e também com o tratamento final dos dados apresentados nas Tabelas 1 e 2, é possível concluir que a cinética enzimática obtida possui comportamento qualitativamente similar ao modelo de Michaelis-Menten, conforme o esperado, e a conversão máxima para a reação de hidrólise do óleo de soja degomado catalisado por lipase de Pseudomonas fluorescens é muito próxima à $90 \%$, com um tempo de reação correspondente a 24 horas. A partir desse resultado, foi possível avaliar o reuso da água e da lipase em sucessivas bateladas, sob as mesmas condições de operação, com tempo de reação de 24 horas.

\subsection{Avaliação do reuso de água e lipase}




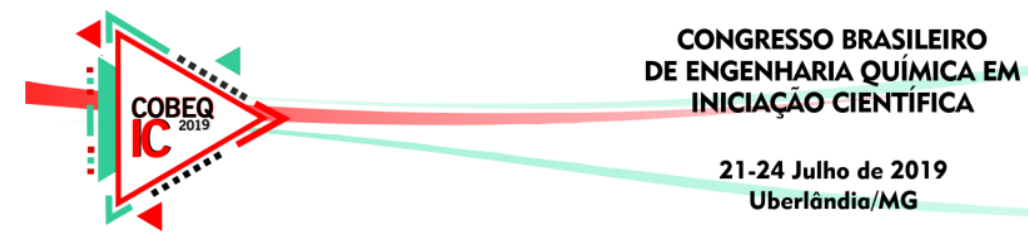

Obtido o tempo de reação para atingir a máxima conversão da reação de hidrólise de óleo de soja degomado catalisado pela lipase de Pseudomonas fluorescens livre, apresenta-se, a seguir, nas Tabelas 3 e 4, referentes aos reatores 1 e 2 em que ocorreu as reações, os dados obtidos em cinco sucessões de bateladas de 24 horas com reuso de água e lipase, e o gráfico de Conversão (\%) vs Batelada com desvio dos dados obtidos, apresentado na Figura 3:

Tabela 3 - Dados obtidos para reuso de lipase e água no Reator 1

\begin{tabular}{|c|c|c|c|c|c|c|c|c|c|c|c|}
\hline \multirow[b]{2}{*}{ Batelada } & \multirow[b]{2}{*}{$\begin{array}{c}\text { Massa } \\
\text { Reator }(\mathrm{g})\end{array}$} & \multicolumn{3}{|c|}{ Branco } & \multicolumn{3}{|c|}{ Amostra } & & 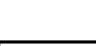 & \multirow[b]{2}{*}{$\begin{array}{c}\text { Conversão } \\
(\%)\end{array}$} \\
\hline & & $\begin{array}{c}\text { Massa } \\
\text { Branco }(\mathrm{g})\end{array}$ & $\begin{array}{l}\mathrm{M} \mathrm{KOH} \\
(\mathrm{mol} / \mathrm{L})\end{array}$ & $\begin{array}{l}\text { V KOH } \\
(\mathrm{mL})\end{array}$ & $\begin{array}{c}\text { Massa } \\
\text { amostra } \\
\text { (g) }\end{array}$ & $\begin{array}{l}\mathrm{M} \mathrm{KOH} \\
(\mathrm{mol} / \mathrm{L})\end{array}$ & $\begin{array}{c}\mathrm{V} \mathrm{KOH} \\
(\mathrm{mL})\end{array}$ & $\begin{array}{c}\text { Massa } \\
\text { Recuperada } \\
\text { (g) }\end{array}$ & $\begin{array}{l}\text { Média } \\
\text { NKOH/g } \\
\text { solução } \\
\text { (mol/g) }\end{array}$ & $\begin{array}{l}\text { Média } \\
\text { branco } \\
\mathrm{NKOH} / \mathrm{g} \\
\text { solução } \\
\text { (mol/g) }\end{array}$ & \\
\hline \multirow{2}{*}{1} & \multirow{2}{*}{149,94} & 1,5391 & 0,0188 & 0,40 & 1,6584 & 0,2409 & 13,40 & \multirow{2}{*}{59,64} & \multirow{2}{*}{$1,98 \mathrm{E}-03$} & \multirow{2}{*}{$4,71 \mathrm{E}-06$} & \multirow{2}{*}{87,63} \\
\hline & & 1,8697 & 0,0188 & 0,45 & 1,8340 & 0,2409 & 15,35 & & & & \\
\hline \multirow{2}{*}{2} & \multirow{2}{*}{183,59} & 1,5245 & 0,0188 & 36,50 & 1,6712 & 0,2409 & 14,40 & \multirow{2}{*}{80,02} & \multirow{2}{*}{$2,02 E-03$} & \multirow{2}{*}{$6,03 E-04$} & \multirow{2}{*}{76,98} \\
\hline & & 1,8168 & 0,2409 & 5,70 & 1,8921 & 0,2409 & 15,90 & & & & \\
\hline \multirow{2}{*}{3} & \multirow{2}{*}{196,75} & 1,6925 & 0,2409 & 5,80 & 1,8872 & 0,2409 & 14,50 & \multirow{2}{*}{112,27} & \multirow{2}{*}{$1,83 \mathrm{E}-03$} & \multirow{2}{*}{$8,20 \mathrm{E}-04$} & \multirow{2}{*}{58,61} \\
\hline & & 1,8043 & 0,2409 & 6,10 & 1,6674 & 0,2409 & 12,50 & & & & \\
\hline \multirow{2}{*}{4} & \multirow{2}{*}{177,13} & 1,9401 & 0,2409 & 5,20 & 1,6349 & 0,2409 & 8,50 & \multirow{2}{*}{74,86} & \multirow{2}{*}{$1,23 \mathrm{E}-03$} & \multirow{2}{*}{$6,19 \mathrm{E}-04$} & \multirow{2}{*}{32,32} \\
\hline & & 1,7923 & 0,2409 & 4,40 & 1,8630 & 0,2409 & 9,40 & & & & \\
\hline \multirow{2}{*}{5} & \multirow{2}{*}{196,37} & 1,6338 & 0,2409 & 4,00 & 1,5924 & 0,2409 & 5,40 & \multirow{2}{*}{110,05} & \multirow{2}{*}{$7,87 \mathrm{E}-04$} & \multirow{2}{*}{$5,90 \mathrm{E}-04$} & \multirow{2}{*}{11,29} \\
\hline & & 1,7960 & 0,2409 & 4,40 & 1,8138 & 0,2409 & 5,70 & & & & \\
\hline
\end{tabular}

Tabela 4 - Dados para o reuso de lipase e água no Reator 2

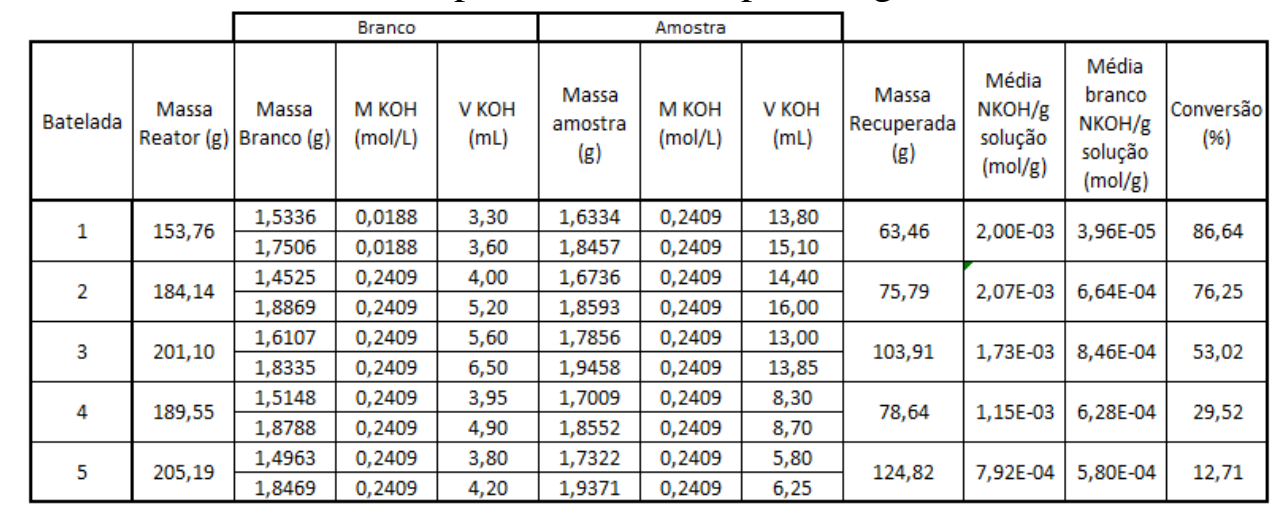

Figura 3 - Gráfico de conversão (\%) da reação de hidrólise de óleo de soja degomado em função do número de reciclos consecutivos de lipase e água

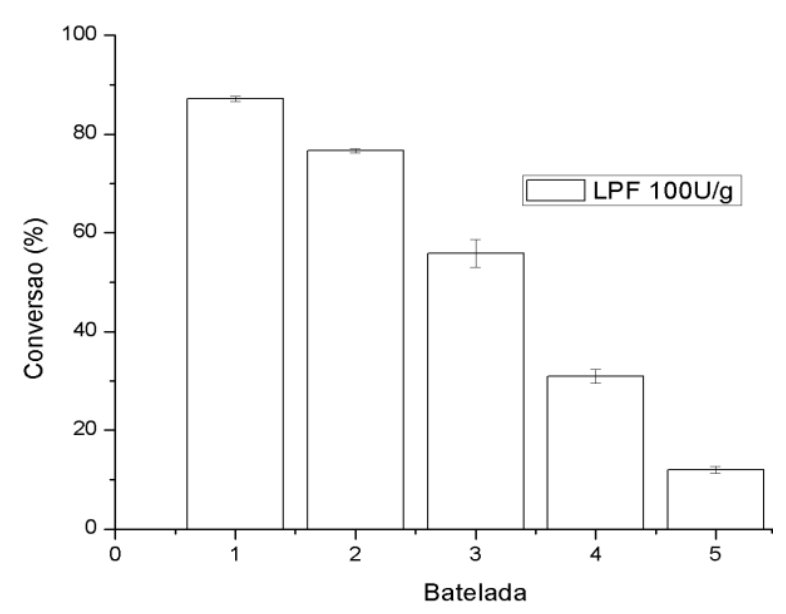




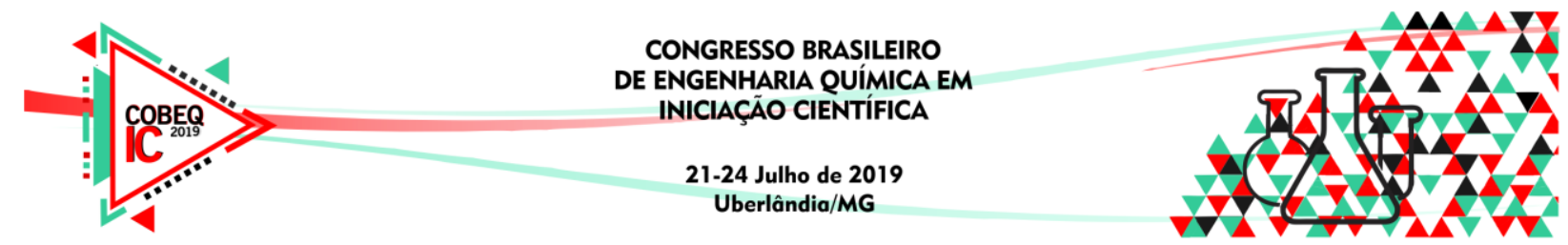

Ao analisar a Figura 3, é possível observar que as primeiras bateladas tiveram um bom rendimento (a primeira teve um valor bem próximo ao obtido no perfil de conversão ao longo do tempo, reforçando a consistência dos resultados), e que a partir da terceira batelada, em que o rendimento é inferior à 50\%, o processo certamente não compensa ser mantido.

Ao final da decantação de cada batelada, observou-se a formação de duas fases, sendo a mais pesada, composta por água, glicerol e lipase, de coloração amarelada clara e mais opaca em relação a fase leve, composta de óleo de soja degomado e ácidos graxos livres. $\mathrm{O}$ esperado para a fase leve é ter uma coloração translúcida, levemente amarronzada (devido à coloração da enzima), o que não foi observável, sugerindo a possibilidade de mono e diglicerídeos criarem uma emulsão com as enzimas, inativando-as conforme o número de bateladas avança e reduzindo a efetividade do reuso das lipases.

\section{CONCLUSÃO}

A partir do estudo do perfil de conversão da hidrólise de óleo de soja degomado catalisado por lipase de Pseudomonas fluorescens livre ao longo do tempo, além do reuso da citada lipase em bateladas sucessivas, é conclusivo que a máxima conversão obtida, de $90 \%$, assim como seu tempo de reação correspondente, de 24 horas, são valores aplicáveis e interessantes para aplicação industrial, visto que se trata de uma enzima na forma livre, solúvel. Em contrapartida, o reuso da lipase não se mostrou promissor, visto que a partir da terceira batelada consecutiva, o rendimento da hidrólise cai consideravelmente, como visto na Figura 3. Dessa forma, buscar alternativas e novas propostas para que o reuso da lipase tenha melhores desempenhos contribuirá para que a produção industrial de biodiesel a partir do óleo de soja degomado esteja mais perto da realidade.

\section{REFERÊNCIAS}

DAVIS, M. E., DAVIS, R. J. Fundamentals of Chemical Reaction Engineering. $1^{\text {st }}$ ed. Boston: McGraw Hill, 2003.

POURZOLFAGHAR, H.; ABNISA, F.; DAUD, W. M. A. W.; AROUA, M. K. A review of the enzymatic hydroesterification process for biodiesel production. Renewable and Sustainable Energy Reviews, 2016, vol. 61, pp 245-257.

ROSSET, D.V., Wancura, J.H.C., UGALDE, G.A. et al. Appl Biochem Biotechnol (2019).

SHULER, M. L., KARGI, F. Bioprocess engineering: basic concepts. 2nd ed. Upper Saddle River, New Jersey: Prentice Hall PTR, 2002.

VESCOVI, V., ROJAS, M.J., BARALDO, A. et al. Lipase-Catalyzed Production of Biodiesel by Hydrolysis of Waste Cooking Oil Followed by Esterification of Free Fatty Acids. Journal of the American Oil Chemists' Society, 2016, vol. 93, pp 1615-1624. 